\title{
Modeling of Electromagnetic Manipulation of Plasmas for Communication During Reentry Flight
}

\author{
Minkwan Kim* and Iain D. Boyd \\ University of Michigan, Ann Arbor, Michigan 48109 \\ and \\ Michael Keidar \\ George Washington University, Washington D.C., 20052 \\ DOI: $\underline{10.2514 / 1.45525}$
}

\begin{abstract}
Radio blackout that occurs during hypersonic reentry flight is an important issue for the operation of the vehicle. Since the radio blackout problem is caused by a high plasma number density around a vehicle, it is necessary to manipulate the plasma to allow communication. We suggest a configuration of an $E \times B$ layer as a reentry blackout mitigation method. The suggested $E \times B$ layer configuration with a two-dimensional magnetic field is simulated using the thermalized potential model and the Poisson-like model in order to illustrate the effectiveness of this approach as a mitigation method. The numerical model uses a magnetohydrodynamics approximation and is solved using a finite volume method with a Riemann solver. The results of the numerical model are assessed using available experimental results. A strong plasma density reduction is obtained when the high electric and magnetic fields are applied near the cathode. The manipulated plasma region provides a possibility to communicate through a plasma layer during a reentry blackout.
\end{abstract}

\section{Nomenclature}

$\mathbf{B}=$ magnetic field, $\mathrm{T}$

$\mathbf{E} \quad=$ electric field, $\mathrm{V} / \mathrm{m}$

$\mathbf{E}_{\perp}=$ electric field across the magnetic field, $\mathrm{V} / \mathrm{m}$

$f_{p}=$ plasma frequency, $\mathrm{Hz}$

$f_{\text {ratio }}=$ radiowave frequency, $\mathrm{Hz}$

$\overline{\mathbf{g}}_{e}=$ relative velocity of electron collisions, $\mathrm{m} / \mathrm{s}$

$\mathbf{J}=$ current density, $\mathrm{A} / \mathrm{m}^{2}$

$m \quad=$ mass, $\mathrm{kg}$

$n=$ plasma number density, $\mathrm{m}^{-3}$

$n_{e} \quad=$ electron number density, $\mathrm{m}^{-3}$

$n_{o}=$ reference plasma number density, $\mathrm{m}^{-3}$

$p \quad=$ pressure, $\mathrm{N} / \mathrm{m}^{2}$

$\mathbf{V}=$ velocity, $\mathrm{m} / \mathrm{s}$

$\beta_{e}=$ electron Hall parameter

$\beta_{x}=x$-direction Hall parameter

$\beta_{z}=z$-direction Hall parameter

$\varepsilon_{0} \quad=$ permittivity of vacuum, $8.5419 \times 10^{-12}[\mathrm{~F} / \mathrm{m}$

$\kappa=$ signal attenuation factor, $\mathrm{dB} / \mathrm{m}$

$\lambda=$ magnetic stream function

$\mu \quad=$ electron mobility, $\mathrm{m}^{2} /(\mathrm{V} \cdot \mathrm{s})$

$v_{c}=$ ion collision frequency, $\mathrm{s}^{-1}$

$v_{e}=$ electron collision frequency, $\mathrm{s}^{-1}$

$\sigma=$ electrical conductivity, $\mathrm{S} / \mathrm{m}$

$\tilde{\sigma}=$ electrical conductivity tensor

$\varphi \quad=$ plasma potential, $\mathrm{V}$

$\varphi^{*} \quad=$ thermalized potential, $\mathrm{V}$

$\omega_{p} \quad=$ plasma frequency, $\mathrm{rad} / \mathrm{s}$

Received 18 May 2009; revision received 20 October 2009; accepted for publication 20 October 2009. Copyright $\odot 2009$ by the American Institute of Aeronautics and Astronautics, Inc. All rights reserved. Copies of this paper may be made for personal or internal use, on condition that the copier pay the $\$ 10.00$ per-copy fee to the Copyright Clearance Center, Inc., 222 Rosewood Drive, Danvers, MA 01923; include the code 0022-4650/10 and $\$ 10.00$ in correspondence with the CCC.

*Graduate Student, Department of Aerospace Engineering; minkwan@ umich.edu. Student Member AIAA.

†Professor, Department of Aerospace Engineering; iainboyd@umich.edu. Associate Fellow AIAA.

¥Assistant Professor of Engineering and Applied Science, Department of Mechanical and Aerospace Engineering; keidar@ gwu.edu. Associate Fellow AIAA.

$$
\begin{aligned}
& \text { Subscripts } \\
& e=\text { electron } \\
& i=\text { ion } \\
& 0=\text { initial value }
\end{aligned}
$$

\section{Introduction}

A HIGH electron number density causes negative effects on a vehicle's operation, particularly for its communications $[1,2]$. Because a plasma layer reflects or attenuates radiowaves that are transmitted from or to a vehicle, communication is temporarily interrupted, which is known as radio blackout. For reentry vehicles, radio blackout typically lasts several minutes, depending on the angle of reentry and the particular trajectory [3] . For example, the Soyuz TMA reentry vehicle experiences about 10 min of radio blackout, and the Mars Pathfinder experienced a $30 \mathrm{~s}$ radio blackout during its Mars entry mission.

Continuous communication and real-time telemetry is very important for manned and unmanned vehicles, for flight safety, catastrophe analysis, and mission success. During $10 \mathrm{~min}$ of radio blackout, the reentry vehicle loses a communication link. It is clearly a flight safety issue for the reentry vehicle. Radio blackout also makes catastrophe analysis impossible, eliminating a critical factor for understanding and preventing reentry accidents. In the Space Shuttle Colombia disaster, telemetry was lost before disintegration, due to radio blackout [4]. There was little available data at the ground station to assist in finding the cause of the disaster. For the unmanned Mars reentry vehicle, a blackout makes the vehicle lose the control or guidance of a control station. In this case, continuous and real-time telemetry determines whether the vehicle succeeds in a mission or not. Therefore, developing solutions that allow communication through a plasma layer is a high priority.

For manned or unmanned reentry vehicles, radio blackout occurs when the plasma frequency of the plasma layer around a vehicle exceeds the radiowave frequency used for communications: $f_{p}>$ $f_{\text {ratio }}$. The plasma frequency $f_{p}$ is calculated from

$$
f_{p}=\frac{\omega_{p}}{2 \pi} \approx 8.9683 \times n_{e}^{1 / 2}(\mathrm{~Hz})
$$

The radio blackout can be solved when the plasma frequency is lower than the radiowave frequency $[3,5]$. In order to lower the plasma frequency, it is necessary to manipulate a plasma number density. 
During the last 50 years, a number of plasma-manipulation approaches have been suggested for mitigation of a plasma layer, including aerodynamic shaping, quenchant injection, high radio frequencies, high transmission power, and Raman scattering. One of the most promising methods is the magnetic-window method [ $\underline{6}-\underline{8}]$, in which a strong de magnetic field is used to allow radiowave propagation through the plasma layer. However, this technique is limited by the required magnetic field strength [9]. The reasonable magnetic field strength is up to $0.15 \mathrm{~T}$ at acceptable weight and volume, but approximately $1 \mathrm{~T}$ magnetic field is required to penetrate the plasma layer by using the magnetic-window method $[8,10]$.

We expand upon the magnetic window via the addition of electric fields to increase the density reduction possible for a given magnetic field strength $[1,3]$. A radiowave can propagate through a plasma layer without disturbing the flow of the plasma or other gases in the system via a window created by an applied $\mathbf{E} \times \mathbf{B}$ field [1]. The applied electric field accelerates the plasma temporarily over the antenna, reducing the local density to allow communication. The mechanism of plasma acceleration across the $\mathbf{E} \times \mathbf{B}$ layer is similar to that in a Hall-effect thruster [1]. The plasma returns to its original velocity and density past the mitigation system. Figure 1 shows a schematic of an applied $\mathbf{E} \times \mathbf{B}$ layer. The use of an electric field alone is impractical, due to shielding of the electrodes by sheath effects. At high densities, the sheath that forms around the electrodes can have a thickness of millimeters or less. However, the presence of a magnetic field can trap the electrons, allowing the existence of a strong electric field. This allows ion acceleration through regions of a plasma layer and corresponding plasma density decrease. The reduced plasma density can create a window in a plasma layer through which radiowaves can be transmitted. The plasma density distribution will be affected by the magnitudes and profiles of the magnetic and electric fields. There are still limits on the density of plasma that can be effected, placed by practical limitations on the magnetic and electric fields that can be applied (due to magnet weight and electrode arcing). However, a significant range of densities can be mitigated, corresponding to increased altitude range of communications or decreased periods of radio blackout. Experimental results confirm our simulation models and numerical results. Therefore, our numerical result can help to estimate the plasma density distribution and establish the optimal $\mathbf{E} \times \mathbf{B}$ layer configuration to allow communication.

In this paper we present a series of models that were developed and compared to experimental data that were conducted to explore the use of electric and magnetic fields to manipulate a plasma layer. This will be seen as an effective approach to solving the blackout problem. First, in Sec. II, we describe a physical model of the plasma and show a fluid plasma approximation. The numerical model of an $\mathbf{E} \times \mathbf{B}$ layer is described in Sec. III, including two types of potential models:

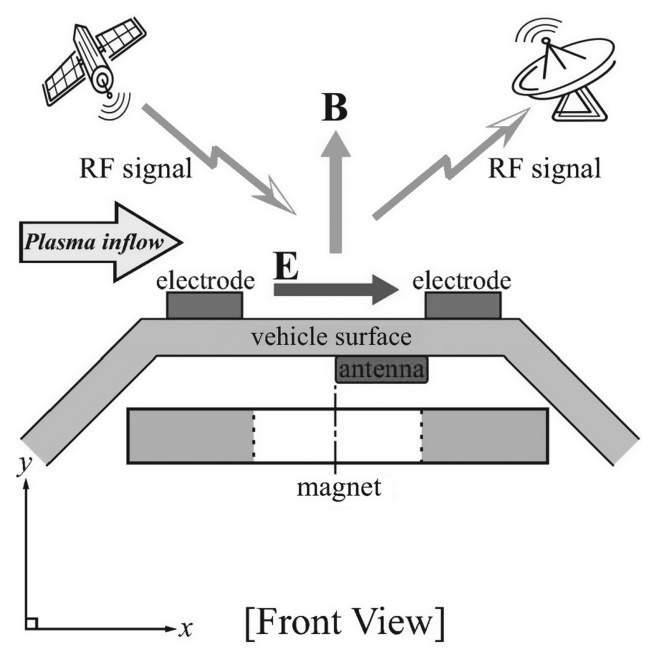

Fig. 1 Schematic of an applied E $\times$ B field for telemetry through a hypersonic plasma layer. a thermalized potential model and a Poisson-like potential model. The numerical and experimental results are compared in Sec. IV.

\section{E x B Layer Model}

We previously developed a two-dimensional magnetohydrodynamics (MHD) model of an $\mathbf{E} \times \mathbf{B}$ layer [3]. This model is based on a two-dimensional steady-state fluid plasma model with the following general assumptions [1]:

1) The $\mathbf{E} \times \mathbf{B}$ layer is quasi-neutral.

2) The neutrals are at rest.

3) There is no ionization in the $\mathbf{E} \times \mathbf{B}$ layer.

4) The electron temperature, $T_{e}$, is constant.

5) The ions are cold.

These assumptions are physically reasonable for this application. First, quasi-neutrality is consistent with measured data from reentry vehicles [11]. Although, strictly speaking, the neutrals are not at rest with an incoming hypersonic flow condition, the stationary neutral assumption will maximize the effect of the ion-neutral drag term in the model, thus rendering our calculations a worst-case scenario [1]. Furthermore, the boundary layer around the vehicle results in relatively slow neutral velocity compared to vehicle velocity [12], and it is in this stagnation layer that the plasma density to be mitigated is highest. The plasma density in the $\mathbf{E} \times \mathbf{B}$ layer decreases due to acceleration. At high hypersonic velocity, electron-impact ionization is more important than associative ionization. Because the electronimpact ionization rate is proportional to the electron number density, the maximum ionization rate corresponds to unperturbed conditions. Therefore, we can assume no ionization in the $\mathbf{E} \times \mathbf{B}$ layer. The coldion assumption makes the ion pressure term negligible. Therefore, the two-dimensional steady-state $\mathbf{E} \times \mathbf{B}$ layer model can be described by

$$
\begin{gathered}
\nabla \cdot\left(\mathbf{V}_{i} n\right)=0 \\
m_{i} n\left(\mathbf{V}_{i} \cdot \nabla \mathbf{V}_{i}\right)=(\mathbf{J} \times \mathbf{B})-\nabla p_{e}-\left(m_{i} n v_{e} \mathbf{V}_{i}+m_{e} n v_{e} \overline{\mathbf{g}}_{e}\right) \\
\mathbf{J}=\sigma\left(\mathbf{E}+\mathbf{V}_{i} \times \mathbf{B}\right)-\frac{\sigma}{e n}(\mathbf{J} \times \mathbf{B}) \\
\nabla \cdot \mathbf{J}=0
\end{gathered}
$$

Equations (2), (3), and (5) are mass, momentum, and current density conservation, respectively, and Eq. (4) is a generalized Ohm's law.

Two-dimensional analysis is important because it can estimate over what range the applied $\mathbf{E} \times \mathbf{B}$ layer can manipulate a hypersonic plasma layer [3]. Previous estimates indicate that the plasma layer is on the order of a few centimeters thick around a reentry vehicle at a density sufficient to block communications $[1,3]$. The region of manipulated plasma must be at least this deep for practical applications. In addition, the magnetic field configuration is an important parameter to optimize the $\mathbf{E} \times \mathbf{B}$ layer configuration. Our twodimensional $\mathbf{E} \times \mathbf{B}$ layer model with a two-dimensional magnetic field uses two different potential distribution models: the thermalized potential model and the Poisson-like model.

\section{A. Thermalized Potential Model}

The magnetic field is assumed to be entirely determined by the applied electromagnet and to be unaffected by the applied electric field. In this case, we can assume that the magnetic field has a divergence-free magnetic field condition. To facilitate the solution of Eq. (4), we can use a magnetic field stream function. The magnetic stream function is calculated from

$$
B_{x}=\frac{\partial \lambda}{\partial z} \quad \text { and } \quad B_{z}=-\frac{\partial \lambda}{\partial x}
$$


The magnetic stream function is constant along a magnetic field line and can be used to discretize the simulation domain in order to reduce it to a quasi-one-dimensional problem.

In the two-dimensional magnetic field condition, the electron mobility is not a simple scalar. In this case, the value of the electron mobility is much larger for electron transport along magnetic field lines than for transport across the magnetic field, which is the primary reason to apply the magnetic field. However, the electron flux cannot be much larger along magnetic field lines than across the magnetic field because of the current conservation. Therefore, along each magnetic field line there is a balance between the electrostatic pressure and the electron thermal pressure [13], and it can be calculated as

$$
-\mu \mathbf{E}_{\perp} n-\mu T_{e} \nabla n=0
$$

Equation (7) can reduce the two-dimensional electric field problem into a quasi-one-dimensional problem, and so it simplifies the electrostatic field calculation. This concept was introduced by Morozov et al. [14] and is known as the thermalized potential or equipotential along the magnetic field line. The thermalized potential $\phi^{*}$ can be defined as

$$
\phi^{*}(\lambda)=\phi(x, z)-T_{e} \ln \left(\frac{n(x, z)}{n_{0}}\right)
$$

\section{B. Poisson-Like Potential Model}

When a high potential drop is applied between two electrodes, the thermalized potential concept is not useful, because a thermalized potential is due to fast electron motion along the magnetic field. In this case, current conservation can be used to calculate an electrostatic field.

The generalized Ohm's law (4 $)$ can be expressed in a matrix form as follows [15]:

$$
\mathbf{J}=\tilde{\sigma}(\mathbf{E}+\mathbf{V} \times \mathbf{B})
$$

The conductivity tensor $\tilde{\sigma}$ is defined as follows [15]:

$$
\tilde{\sigma}=\frac{\sigma}{1+\beta^{2}}\left[\begin{array}{cc}
1+\beta_{x}^{2} & \beta_{x} \beta_{z} \\
\beta_{x} \beta_{z} & 1+\beta_{z}^{2}
\end{array}\right]
$$

where

$$
\beta_{x}=B_{x} \frac{\beta_{e}}{B} \quad \text { and } \quad \beta_{z}=B_{z} \frac{\beta_{e}}{B}
$$

From Eqs. (5) and (9) we can obtain a Poisson-like equation for the potential distribution:

$$
\nabla \cdot(\tilde{\sigma} \nabla \phi)=\nabla \cdot(\mathbf{V} \times \mathbf{B})
$$

Equation (12) is a nonlinear second-order elliptic partial differential equation that gives the potential distribution for a high-potential-drop condition.

\section{Numerical Method}

As shown in Fig. 2, the two-dimensional $\mathbf{E} \times \mathbf{B}$ layer MHD model is solved numerically with an iterative scheme. The solution begins with an assumed initial potential distribution $\varphi^{0}$, which is obtained by solving the Poisson equation (12). Equations (2) and (3), the ion transport model, are solved by using the finite volume method with the Harten-Lax-van Leer contact wave Riemann solver $[16,17]$ to give plasma number density and velocity. The obtained plasma number density and velocity distributions are used to calculate the new potential distribution. The potential distribution is obtained from either the thermalized potential model or the Poisson-like potential model, depending on the strength of the applied electric field.

The new potential distribution provides a new electric field for the next iteration step. After several iterations, all variables converge and we obtain a steady-state solution with sufficient accuracy.

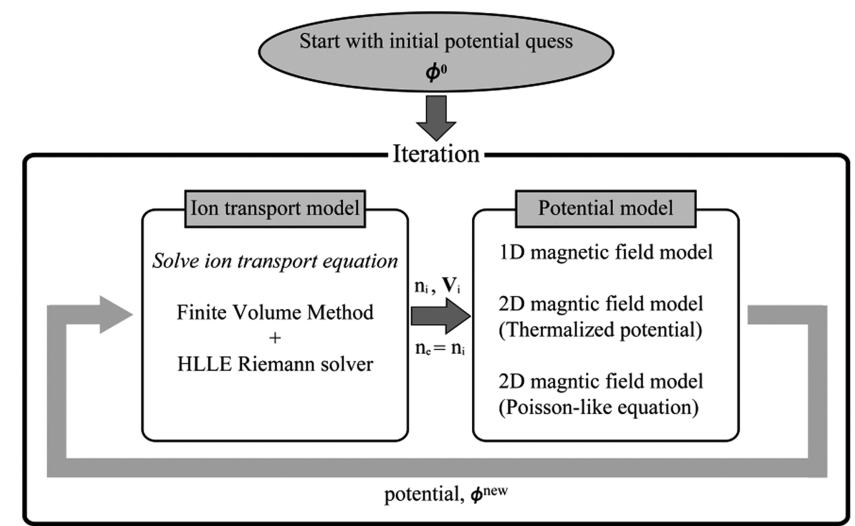

Fig. 2 Numerical method for a two-dimensional $E \times$ B layer MHD model.

Figure 3 shows the boundary conditions that are applied in the two-dimensional $\mathbf{E} \times \mathbf{B}$ layer MHD model. The inflow boundary condition is applied on the left side. It uses the initial plasma number density and the constant neutral number density. In this case, the bulk plasma velocity is assumed to be $1000 \mathrm{~m} / \mathrm{s}$ with only an $x$-direction component. The right- and upper-side boundaries use the outflow boundary condition, and a zero potential variation across the boundary is assumed. The bottom boundary uses the dielectric boundary condition. Because of the dielectric boundary condition, no ions are neutralized at the dielectric wall, which means that the wall fully absorbs ions.

Figure 4 shows a configuration of an applied magnetic field [18]. The configuration of a magnetic field does not depend on the maximum magnetic field strength. The maximum magnetic field strength is given near the two electrodes.

\section{Results}

\section{A. Numerical Simulation Results}

The plasma density reduction with the two-dimensional magnetic field is shown in Fig. 5 , in which the initial plasma density $n_{0}$ is $4.2 \times 10^{16} \mathrm{~m}^{-3}$ and the initial neutral pressure is 1 mtorr. A $-800 \mathrm{~V}$ potential is applied with $0.07 \mathrm{~T}$ magnetic field strength [18]. It has two increased density regions beyond the anode and the cathode. The increased density region of the cathode is also found in the one-dimensional constant-magnetic-field model [3]. It is primarily caused by the $0 \mathrm{~V}$ potential beyond the cathode. The applied $0 \mathrm{~V}$ potential is quite reasonable, because we assume that the area beyond the cathode has a vacuum condition. However, the one-dimensional

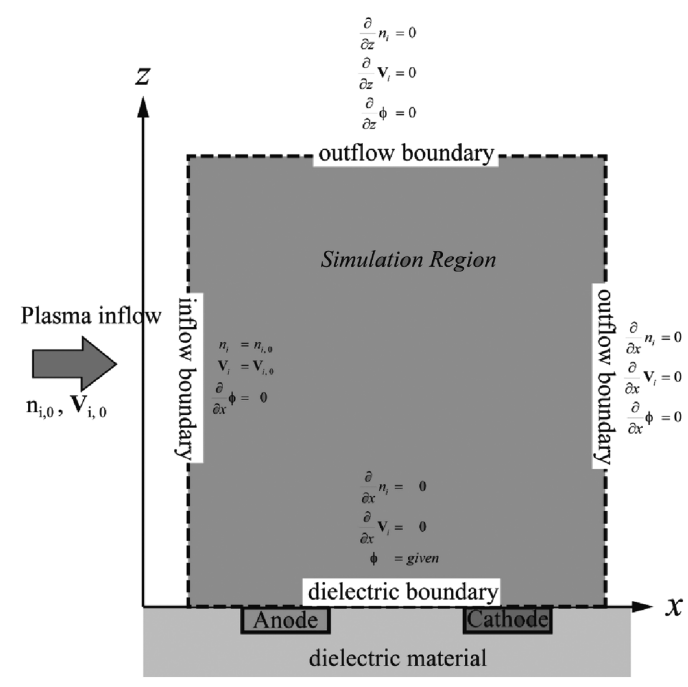

Fig. 3 Applied boundary conditions for a two-dimensional E $\times$ B layer model. 

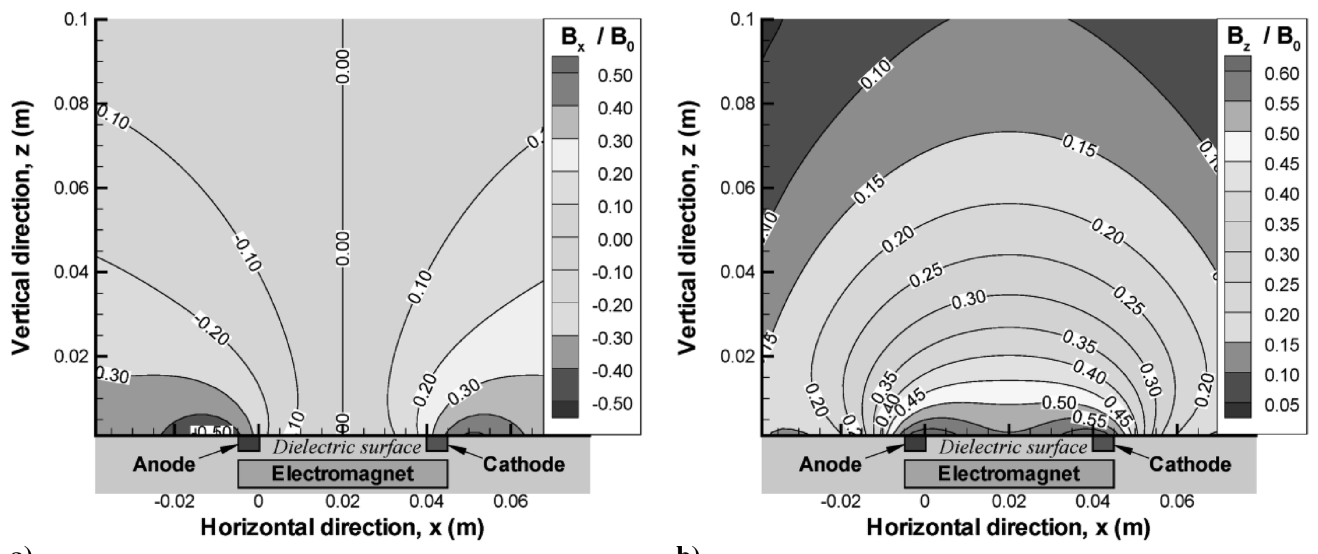

a)

b)

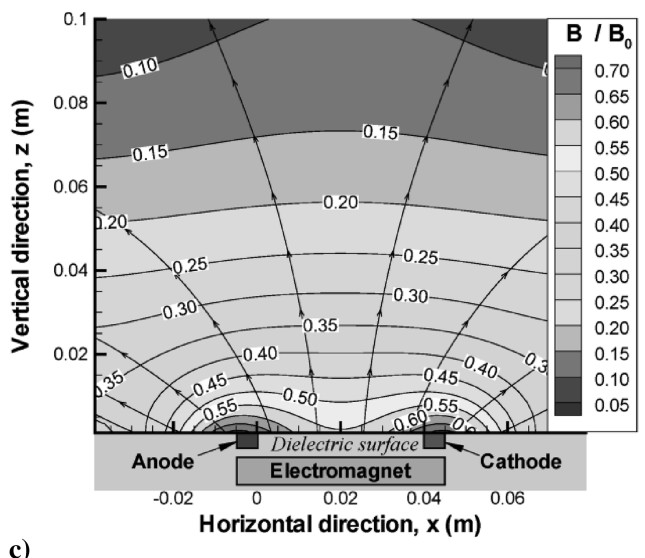

Fig. 4 Applied magnetic field configuration normalized by the maximum magnetic field strength: a) horizontal direction magnetic field configuration, $\left.B_{x} / B_{\max } ; b\right)$ vertical direction magnetic field configuration, $B_{z} / B_{\max }$; and c) normalized magnetic field strength configuration with the magnetic field lines.

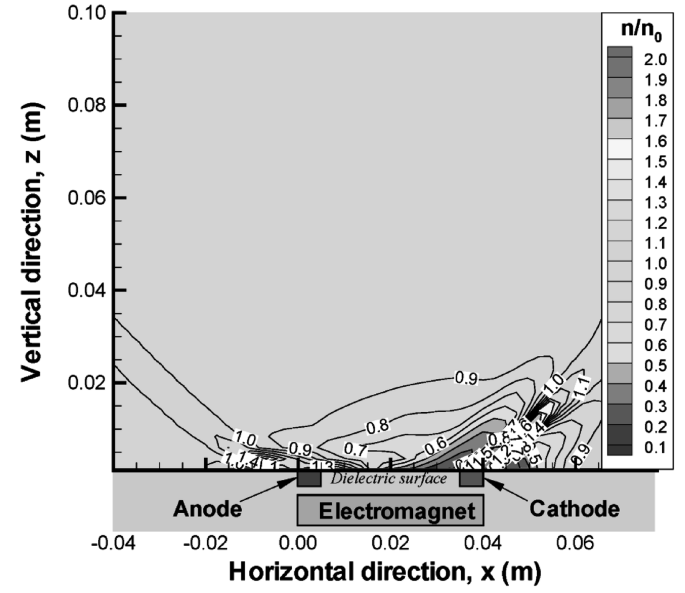

Fig. 5 Distribution of plasma-density-reduction ratio.

magnetic field model does not have the increased density region near the anode [1]. This increased region is due to the divergence of the applied two-dimensional magnetic field.

Figure 6 shows more detail of the plasma-density-reduction profile. The highest plasma density reduction occurs near the cathode and the $\mathbf{E} \times \mathbf{B}$ layer can effectively reduce the plasma density up to approximately $2 \mathrm{~cm}$ above the surface. Therefore, such analysis can determine the optimal location of the transmit antenna to solve the radio blackout.

It is important to understand the effectiveness of the applied magnetic field in the $\mathbf{E} \times \mathbf{B}$ layer in order to optimize the system. In a previous study, we showed that a stronger magnetic field gives a higher plasma density reduction with the one-dimensional $\mathbf{E} \times \mathbf{B}$ layer MHD model [1]. The two-dimensional $\mathbf{E} \times \mathbf{B}$ layer MHD model also gives similar results. Figure 7 shows the plasma density reduction in the $\mathbf{E} \times \mathbf{B}$ layer with several magnetic field strengths. In those cases, we use one-dimensional constant-magnetic-field distribution. As expected, a higher magnetic field gives a higher density reduction. However, the maximum magnetic field strength is limited because of the weight of a permanent magnet. The higher magnetic field guarantees a larger window for communication but it gives a negative effect on vehicle performance due to a heavy weight.

As shown in Fig. 8, the one-dimensional magnetic field model predicts a higher plasma-density-reduction ratio than the two-

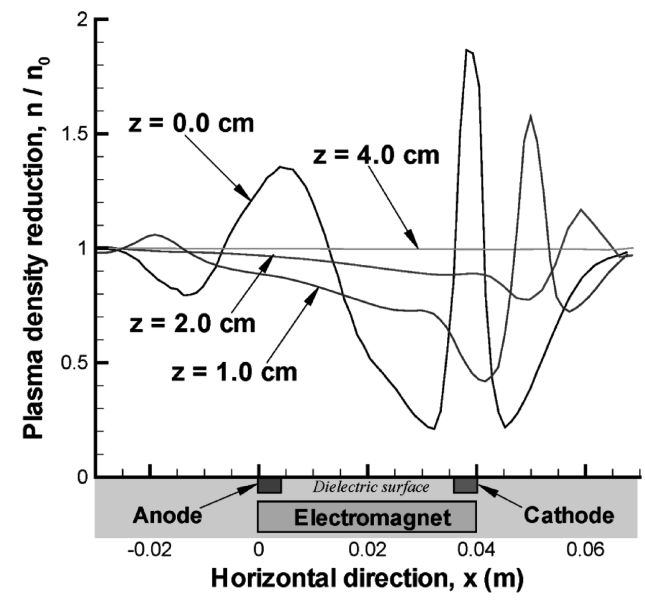

Fig. 6 Distributions of the plasma-density-reduction ratios at several vertical positions which are $z=0.0,1.0,2.0$, and $4.0 \mathrm{~cm}$. 


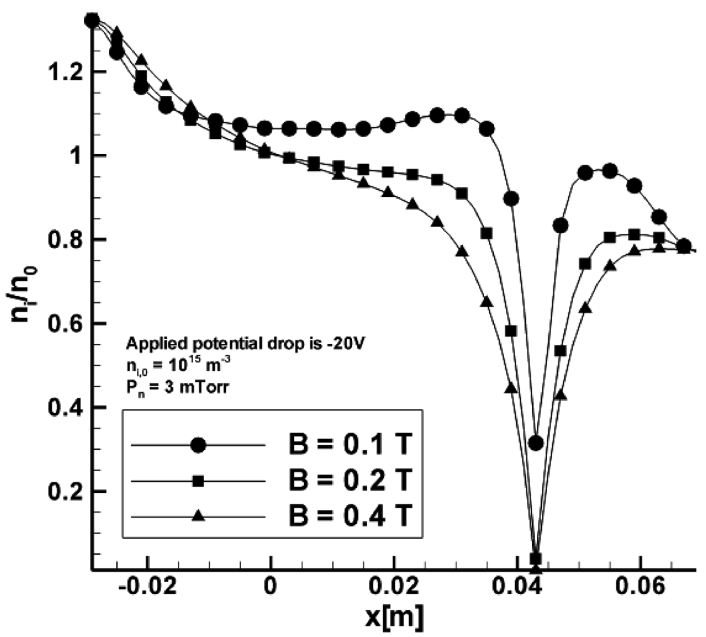

Fig. 7 Plasma density profiles with several magnetic field strengths.

dimensional magnetic field model. Therefore, we can expect the three-dimensional magnetic field will give a smaller plasma density reduction than the two-dimensional magnetic field. Because the real magnetic field has a three-dimensional distribution, the plasma density reduction of an $\mathbf{E} \times \mathbf{B}$ layer will be less than the predicted plasma density reduction by the two-dimensional magnetic field. Figure 8 also shows the effectiveness of the magnetic field strength with one-dimensional and two-dimensional $\mathbf{E} \times \mathbf{B}$ layer MHD models. The plasma-density-reduction ratio increases with magnetic field strength. However, the increase of the plasma density reduction becomes smaller at the strong magnetic field condition. The $0.2 \mathrm{~T}$ magnetic field condition gives the maximized plasma-densityreduction ratio. It is also interesting that all models are agreement in the case of large magnetic field, stronger than $0.2 \mathrm{~T}$.

The potential drop is another parameter that affects the plasma density reduction in the applied $\mathbf{E} \times \mathbf{B}$ layer. In the one-dimensional case, we showed that a higher potential drop gives a higher plasma density reduction but the maximum applicable potential drop is limited due to a technical limitation [1]. For the two-dimensional $\mathbf{E} \times \mathbf{B}$ layer MHD model, the effectiveness of the applied potential drop is shown in Fig. 9. In this case, the initial plasma density, $n_{0}$ is $4.2 \times 10^{16} \mathrm{~m}^{-3}$ and the initial neutral pressure is $1 \mathrm{mTorr}$ with $0.07 \mathrm{~T}$ magnetic field strength.

\section{B. Comparisons and Analysis}

Figure shows both experimental results [18] and one-dimensional magnetic field model results where the initial plasma density, $n_{0}$ is $4.2 \times 10^{16} \mathrm{~m}^{-3}$, the initial neutral pressure is $1 \mathrm{mTorr}$ and a $-800 \mathrm{~V}$ potential is applied with $0.07 \mathrm{~T}$ magnetic field strength. The vertical

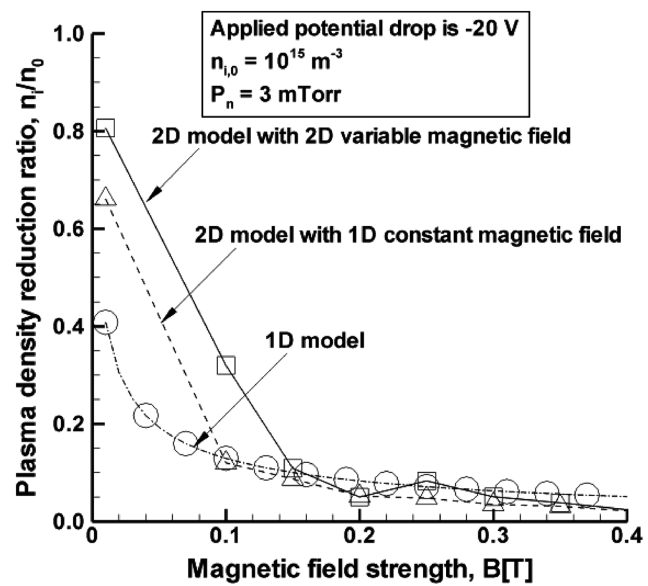

Fig. 8 Plasma-density-reduction ratio as a function of magnetic field with three different models.

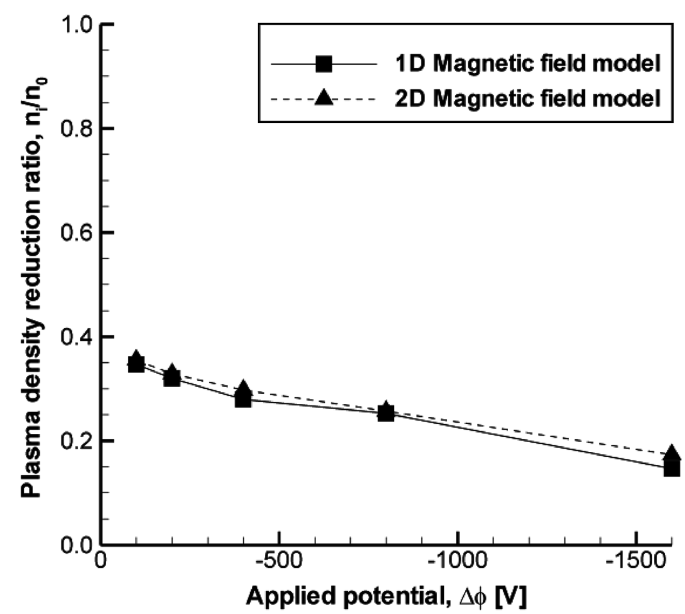

Fig. 9 Plasma-density-reduction ratio as a function of the applied potential drop.

and horizontal locations are normalized by the interelectrode length, $L$. The anode and cathode are located at $x / L=0$ and $x / L=1.0$, respectively. The simulations use a $200 \times 300$ structured rectangular grid. As shown in Fig. 10, the $\mathbf{E} \times \mathbf{B}$ layer model agrees well with the measured data near the center of the two electrodes (see Fig. 10b). However, the model usually predicts higher plasma-densityreduction ratios than the experimental results at the $x / L=0.675$ and 1.0 positions. The model uses the one-dimensional magnetic field condition, which means the magnetic field only has a constant $z$-direction component. The experimental magnetic field is not onedimensional except at the center of the two electrodes, $x / L=0.5$ position. The two-dimensional magnetic field model gives a better result than the one-dimensional magnetic field model as shown in Fig. 11. In this case, the initial conditions and the $\mathbf{E} \times \mathbf{B}$ layer configuration are same as Fig. 10.

In Figs. 10 and 11, the numerical result does not include a generation of a plasma layer so the entire simulation domain is assumed as a plasma layer. It is a reasonable assumption in order to compare with the experimental data because the experimental test chamber is completely filled with Helicon plasma. The thickness of a plasma layer is same as the radius of a test chamber and it is typically thicker than a plasma layer of a reentry flight. For the RAM-C flight test, the thickness of a plasma layer is approximately $1-2 \mathrm{~cm}$ but it depends on the altitude. For the low-altitude case, the plasma layer is thin and has high peak plasma density. At high altitudes, the plasma layer is thicker and the peak plasma density is lower than the lowaltitude case $[\underline{9}, 19]$. Therefore, it has two important values in order to determine a possibility of solving a radio blackout problem, the reduced plasma number density and the thickness of plasma layer which is effectively manipulated by an $\mathbf{E} \times \mathbf{B}$ layer.

The reduced plasma number density is one of important value in order to verify a possibility of a communication through a plasma layer by using an $\mathbf{E} \times \mathbf{B}$ layer. When a plasma number density is higher than a critical number density, a radiowave has an infinite attenuation in a plasma layer $[\underline{3}, \underline{20}]$. For $1 \mathrm{GHz}$ ultra-high-frequency radiowave, the reduced plasma number density by an $\mathbf{E} \times \mathbf{B}$ layer at least should be less than $4.0 \times 10^{16} \mathrm{~m}^{-3}$ [21]. Even if the plasma number density is less than the critical plasma number density, there is still radiowave attenuation. In this case, the radiowave attenuation mainly depends on the electron number density [3]. Therefore, a desired plasma number density should be considered with radiowave frequency and maximum signal attenuation for a communication.

As shown in Figs. 10 and 11, the high plasma density reduction occurs near the cathode, and the plasma density reduction can be extended up to $4 \mathrm{~cm}$ from the bottom surface. This demonstrates how far the applied $\mathbf{E} \times \mathbf{B}$ layer can effectively reduce the plasma density from the surface. The area over which the applied $\mathbf{E} \times \mathbf{B}$ layer can effectively reduce the plasma density is very important, because it should be larger than the plasma density peak location of the hypersonic plasma layer in order to solve the radio blackout. The 

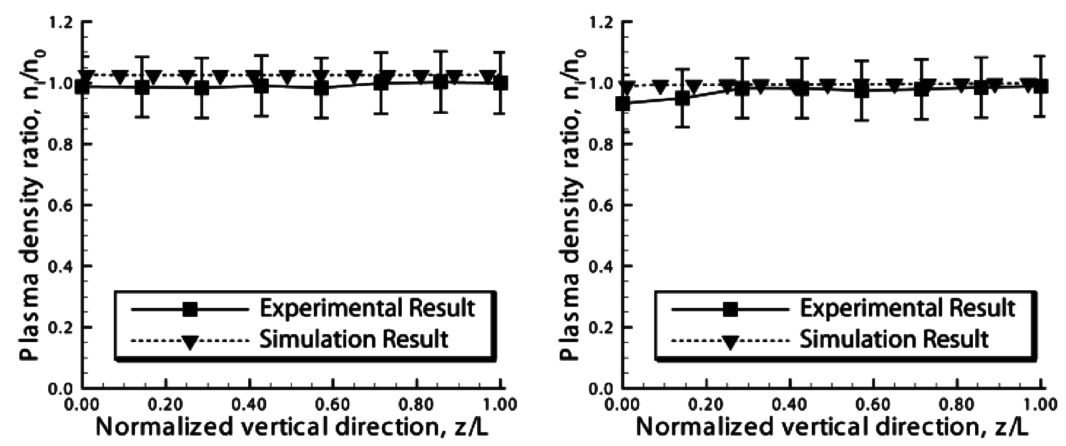

a) $\mathrm{x} / \mathrm{L}=\mathbf{0 . 0 2 5}$

b) $\mathrm{x} / \mathrm{L}=\mathbf{0 . 3 5}$
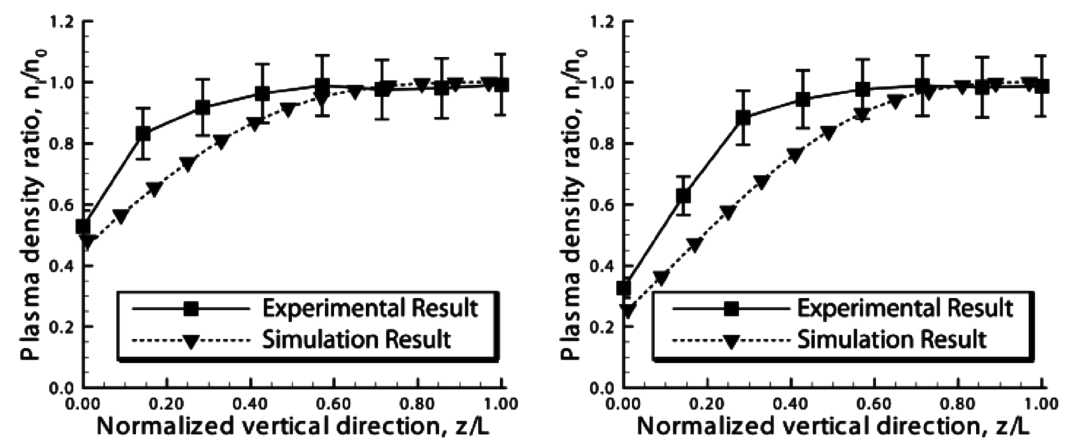

c) $x / L=0.675$

d) $\mathbf{x} / \mathrm{L}=1.0$

Fig. 10 Plasma density profiles at several locations from experiment and simulation (using the one-dimensional magnetic field configuration).

plasma density peak typically occurs at $1-2 \mathrm{~cm}$ above the vehicle surface in the RAM-C flight test [1,19], and so the applied $\mathbf{E} \times \mathbf{B}$ layer can penetrate a plasma layer for communication during a radio blackout period.

The previous passive plasma-manipulation approaches affect the aerodynamic performance of a vehicle, such as drag and heat transfer [9]. Figures $\underline{10}$ and $\underline{11}$ also show that the applied $\mathbf{E} \times \mathbf{B}$ layer only affects the plasma density near the cathode because of a potential distribution, as shown in Fig. 12. The potential sharply changes near the cathode, and so it gives a strong electric field for ion acceleration near the cathode, which means an $\mathbf{E} \times \mathbf{B}$ layer will not disturb an entire flow. This is an important fact for a practical application, because an applied $\mathbf{E} \times \mathbf{B}$ layer gives a window for communication without any effect on a vehicle's aerodynamics.
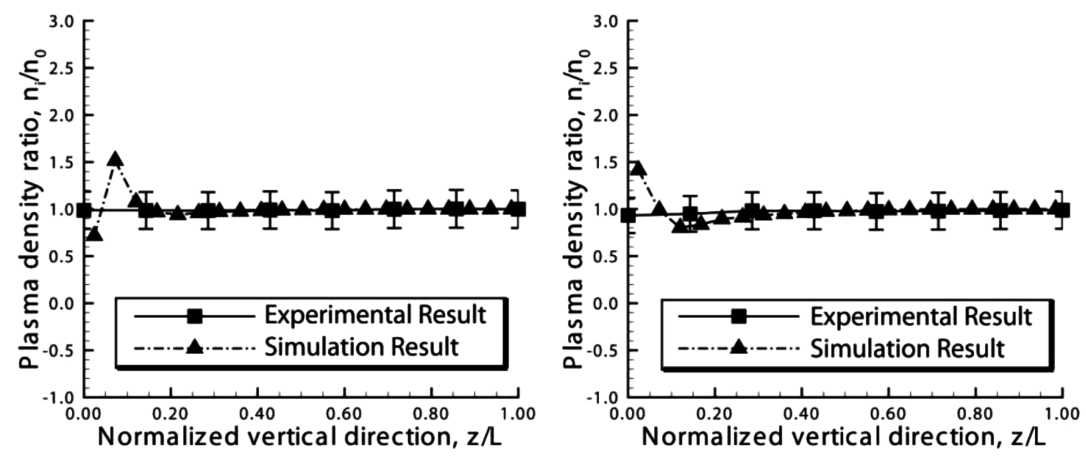

a) $\mathbf{x} / \mathrm{L}=\mathbf{0 . 0 2 5}$

b) $\mathrm{x} / \mathrm{L}=\mathbf{0 . 3 5}$
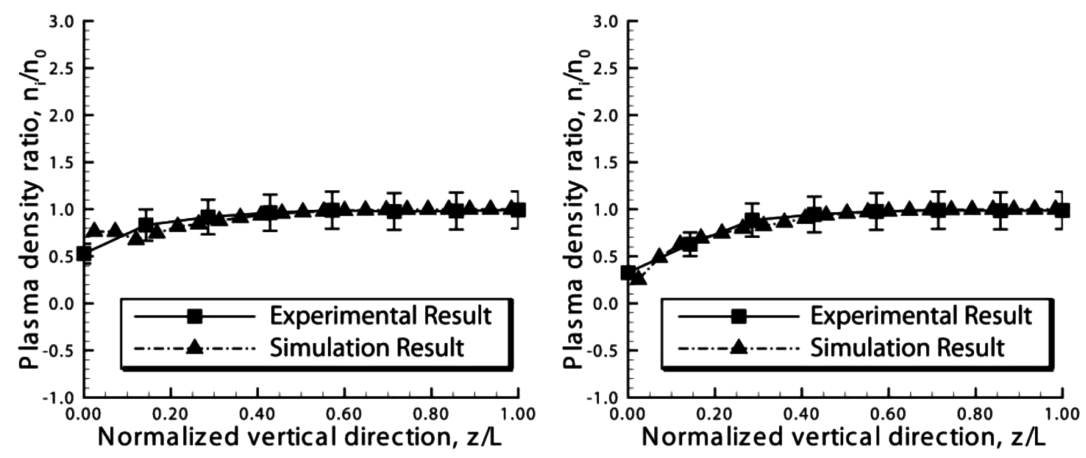

c) $\mathrm{x} / \mathrm{L}=\mathbf{0 . 6 7 5}$

d) $\mathrm{x} / \mathrm{L}=\mathbf{1 . 0}$

Fig. 11 Plasma density profiles at several locations from simulation and experiment with a two-dimensional magnetic field. 


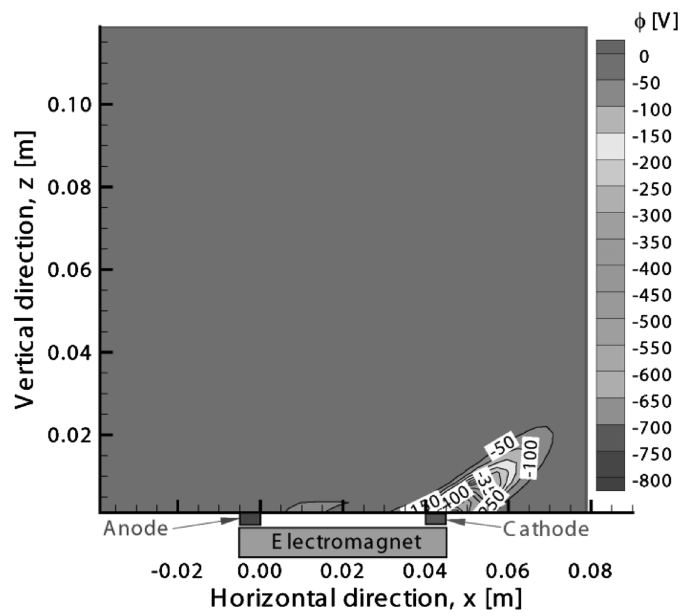

Fig. 12 Potential distribution with a two-dimensional magnetic field.

\section{Conclusions}

We have studied an $\mathbf{E} \times \mathbf{B}$ layer to allow communication through the plasma layer during the radio blackout period. The numerical result shows a significant density reduction in an $\mathbf{E} \times \mathbf{B}$ layer. The application of electric and magnetic fields could therefore allow radio communication through a hypersonic plasma layer. The numerical result also shows that the applied $\mathbf{E} \times \mathbf{B}$ layer reduces a plasma number density near a cathode. The reduced plasma density and area can be controlled by changing magnetic field strength and potential. Because the applied $\mathbf{E} \times \mathbf{B}$ layer can manipulate plasma in a specific region, it can also be used in other industry applications such as semiconductor production and positron storage.

\section{Acknowledgments}

The authors gratefully acknowledge the support of the U.S. Air Force in funding this work. The authors wish to thank Dave Morris, Kristina Lemmer, Alec Gallimore, Brian Gilchrist, Peter Peterson, Tim Smith, Jonathan Zagel, Kenneth G. Powell, and Charles Jones for very useful discussions on this subject.

\section{References}

[1] Keidar, M., Kim, M., and Boyd, I. D., "Electromagnetic Reduction of Plasma Density During Atmospheric Reentry and Hypersonic Flights," Journal of Spacecraft and Rockets, Vol. 45, No. 3, May-June 2008, pp. $445-453$. doi: $10.2514 / 1.32147$

[2] Hodara, H., "The Use of Magnetic Fields in the Elimination of the Reentry Radio Blackout," Proceedings of the IRE, Vol. 49, No. 12, Dec. 1961 , pp. $1825-1830$. doi:10.1109/JRPROC.1961.287709

[3] Kim, M., Keidar, M., Boyd, I. D., "Analysis of an Electromagnetic Mitigation Scheme for Reentry Telemetry Through Plasma," Journal of Spacecraft and Rockets, Vol. 45, No. 6, Nov.-Dec. 2008, pp. 12231229.

doi: $10.2514 / 1.37395$

[4] "Columbia Accident Investigation Board Report," Vol. 1, Government Printing Office, Washington, D.C., 2003, Chap. 2, http://caib1.nasa. gov/news/report/volume1/ [retrieved 20 May 2009].

[5] Rybak, J. P., and Churchill, R. J., "Progress in Reentry Communications," IEEE Transactions on Aerospace and Electronic Systems,
Vol. aes-7, No. 5, 1971, pp. 879-894. doi:10.1109/TAES.1971.310328

[6] Rawhouser, R., "Overview of the AF Avionics Laboratory reentry Electromagnetics Program," The Rntry Plasma Sheath and its Effects on Space Vehicle Electromagnetic Systems, Vol. I, NASA Langley Research Center NASA SP-252, 1964, pp. 3-17.

[7] Spencer, D. F., "An Evaluation of the Communication Blackout Problem for a Blunt Mars-Entry Capsule and a Potential Method for the Elimination of Blackout," Jet Propulsion Lab. TR 32-594, Pasadena, CA, April 1964.

[8] Hartunian, R. A., Stewart, G. E., Curtiss, T. J., Fergason, S. D., and Seibold, R. W., "Implications and Mitigation of Radio Frequency Blackout During Reentry of Reusable Launch Vehicles," AIAA Paper 20076633, 2007

[9] Hartunian, R. A., Stewart, G. E., Fergason, S. D., Curtiss, T. J., and Seibold, R. W., "Causes and Mitigation of Radio Frequency (RF) Blackout During Reentry of Reusable Launch Vehicles," The Aerospace Corp., Rept. ATR-2007 (5309)-1, El Segundo, CA, Jan. 2007.

[10] Starkey, R., Lewis, R., and Jones, C., "Electromagnetic Wave/Magnetoactive Plasma Sheath Interaction for Hypersonic Vehicle Telemetry Blackout Analysis," 34th AIAA Plasmadynamics and Lasers Conference, AIAA Paper 2003-4167, June 2003.

[11] Sims, T. E., and Jones, R. F., "Flight Measurements of VHF-Signal Attenuation and Antenna Impedance for the RAM A-1 Slender Probe at Velocities up to 17,800 Feet per Second," NASA TM X-760, 1963.

[12] Li, X., Fu, D., and Ma, Y., "Direct Numerical Simulation of Hypersonic Boundary-Layer Transition over a Blunt Cone," AIAA Journal, Vol. 46, No. 11, 2008, pp. 2899-2913. doi: $10.2514 / 1.37305$

[13] M. Keidar, Boyd, I. D., and, Beilis, I. I., "Plasma Flow and Plasma-Wall Transition in Hall Thruster Channel," Physics of Plasmas, Vol. 8, No. 12, 2001, pp. 5315-5322. doi:10.1063/1.1421370

[14] Morozov, A. I., Esipchuk, Yu. V., Tilinin, G. N., Trofimov, A. V., Sharov, Yu. A., and Shchepkin, G. Ya., "Plasma Accelerator with Closed Electron Drift and Extended Acceleration Zone," Soviet Journal of Plasma Physics, Vol. 17, No. 1, 1972, pp. 38-45.

[15] Borghi, C. A., Carraro, M. R., and Cristofolini, A., "Numerical Modeling of MHD Interaction in the Boundary Layer of Hypersonic Flows," IEEE Transactions on Magnetics, Vol. 39, No. 3, May 2003, pp. 1507-1510. doi:10.1109/TMAG.2003.810416

[16] Colella, P., Dorr, M. R., and Wake, D. D., "A Conservative Finite Difference Method for the Numerical Solution of Plasma Fluid Equations," Journal of Computational Physics, Vol. 149, No. 1, 1999, pp. 168-193. doi: $10.1006 /$ jcph.1998.6136

[17] Li, S., "An HLLC Riemann Solver for Magneto-Hydrodynamics," Journal of Computational Physics, Vol. 203, No. 1, 2005, pp. 344-357. doi:10.1016/j.jcp.2004.08.020

[18] Lemmer, K. M., Gallimore, A. G., Smith, T. B., Davis, C. N., and Peterson, P., "Experimental Results from Application of an $\mathbf{E} \times \mathbf{B}$ Layer for Investigation of Communications Blackout Amelioration," Journal of Spacecraft and Rockets, Vol. 46, No. 6, Nov. 2009, pp. 1100 1109.

[19] Jones, W. L., and Cross, A. E., "Electrostatic Probe Measurements of Plasma Surrounding Three 25,000 Foot per Second Reentry Flight Experiments," NASA SP-252, 1970.

[20] Rybak, J., and Churchill, R., "Progress in Reentry Communications," IEEE Transactions on Aerospace and Electronic Systems, Vol. aes-7, No. 5, Sept. 1971, pp. 879-894. doi:10.1109/TAES.1971.310328

[21] Kim, M., Keidar, M., and Boyd, I. D., "Two-Dimensional Model of an Electromagnetic Layer for the Mitigation of Communications Blackout," AIAA Paper 2009-1232, Jan. 2009.

A. Ketsdever Associate Editor 